\title{
LA COMPLEJIDAD DE LA NOÉTICA EN MATEMÁTICAS COMO CAUSA DE LA FALTA DE DEVOLUCIÓN
}

Bruno D’ Amore*

\begin{abstract}
This study derives inspiration from the original discussions of Raymond Duval (1988 $a, b, c ;$ 1993), and forms part of the research being done by the NRD of Bologna University. It attempts to draw out and to substantiate the differences hypotheses that lie at the foundations of unsuccessful devolution (Perrin Glorian, 1994), and therefore also at the foundations of the schooling of mathematical awareness (D'Amore, 1999a)
\end{abstract}

\section{RESUMEN}

Este trabajo se inspira en los estudios en los que ha sido pionero indiscutible Raymond Duval (1988 a,b,c; 1993), y se sitúa en la línea de investigación del NRD de Bolonia, que busca localizar y evidenciar las diferentes hipótesis que se hallan en la base de la falta de devolución (Perrin Glorian 1994), y por lo tanto en la base de la escolarización del saber matemático (D’Amore 1999a).

Palabras claves: Registros semióticos, Noética, semiótica, devolución.

\section{Concepto y objeto en matemáticas}

Cada concepto en matemáticas:

- Tiene reenvíos a "no-objetos"; por tanto la conceptualización no es y no puede ser basada sobre significados que se apoyan sobre la realidad concreta; en otras palabras en matemáticas no son posibles reenvíos ostensibles.

- Esta obligado a utilizar representaciones, dado que estos no son "objetos reales" de exhibirse en lugar del concepto o en su evocación; por tanto la conceptualización debe necesariamente pasar a través de registros representativos que, por varios motivos,

\footnotetext{
* N.R.D. Núcleo de investigación en Didáctica de la Matemática. Departamento de Matemática. Universidad de Bologna. Italia.
} 
sobretodo si son de carácter lingüístico, no pueden ser unívocos [aquí "objeto real" esta entendido en el sentido intuitivo de "cosa". El significado de ésta, está bien explicado en la Metafísica de Aristóteles, cuando afirma que la "cosa", en cuanto parte de la realidad, es todo lo que presenta las siguientes tres características:

- Tridimensionalidad.

- Accesibilidad sensorial múltiple (es decir por más de un sentido a la vez) independente de las representaciones semióticas.

— Posibilidad de separación material de otras partes de la realidad, de otras "cosas"].

- Se habla más específicamente en matemáticas de "objetos matemáticos" que no de conceptos matemáticos en cuanto que en matemáticas se estudian preferiblemente los objetos que no los conceptos (Duval, 1998).

En Duval la noción de concepto, preliminar o de cualquier forma prioritaria en casi todos los Autores, se presenta secundaria, mientras la que asume un carácter de prioridad es la pareja: sistema de signos-objeto, como pondré en evidencia en el momento en el cual haré referimento a la paradoja cognitiva del pensamiento matemático, evidenciado por el mismo Duval (1993, pág. 38).

Mucho más que un discurso de palabras, me parece que puede ser más eficaz el siguiente esquema:

"objeto matemático a conceptualizar: no existe como objeto real

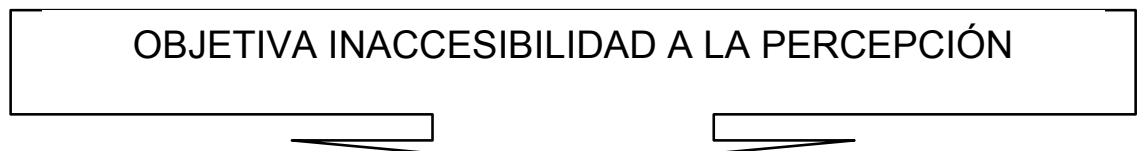

(consecuente necesidad de) representaciones semióticas

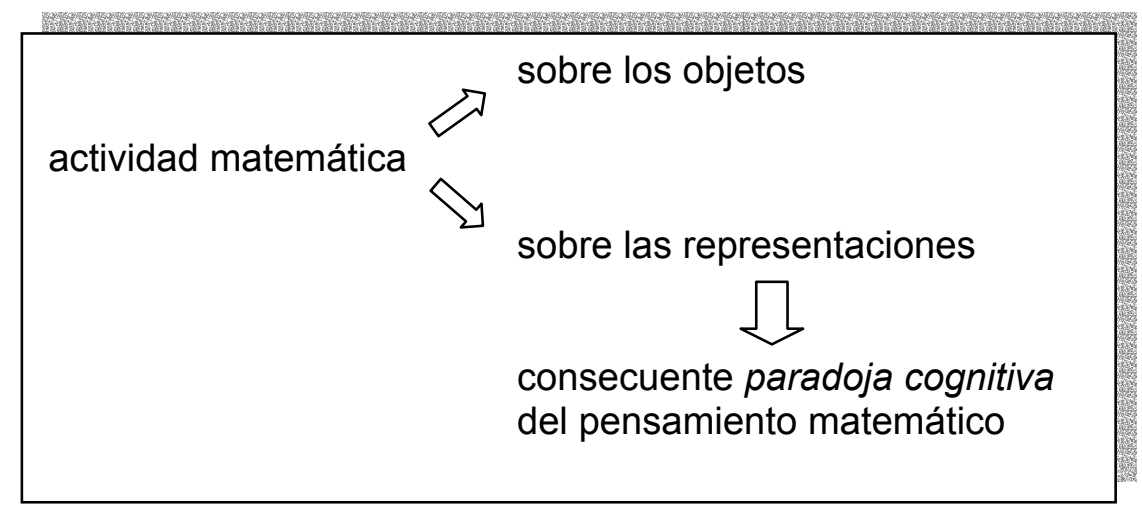

\section{La paradoja cognitiva del pensamiento matemático}

Veamos en que consiste esta paradoja (Duval, 1993, pág. 38; la traducción es mía, concordada con el Autor): 
“(..) de una parte, el aprendizaje de los objetos matemáticos no puede ser sino un aprendizaje conceptual y, de otra parte, es solo por medio de representaciones semióticas que es posible una actividad sobre los objetos matemáticos. Esta paradoja puede constituir un verdadero círculo vicioso para el aprendizaje. ¿Cómo, quienes están en fase de aprendizaje podrían no confundir los objetos matemáticos con sus representaciones semióticas siendo que ellos no pueden tener relación más que con las representaciones semióticas? La imposibilidad de un acceso directo a los objetos matemáticos, diferente de cada representación semiótica, hace la confusión casi inevitable. Y, al contrario, ¿cómo pueden ellos adquirir dominio de los tratamientos matemáticos, necesariamente ligados a las representaciones semióticas, si no tienen ya un aprendizaje conceptual de los objetos representados? Esta paradoja es aún más fuerte si se identifica actividad matemática con actividad conceptual y si se consideran las representaciones semióticas como secundarias o extrínsecas".

Tan solo por claridad terminológica, pero sin pretender abarcar todo, dado que no siempre estos términos son usados en el mismo sentido, prefiero explicar los significados y los símbolos de los cuales me sirvo a continuación:

semiótica $={ }_{\mathrm{df}}$ adquisición de una representación realizada por medio de signos noética $=_{\text {df }}$ adquisición conceptual de un objeto

Entenderé, de ahora en adelante:

$r^{m}={ }_{d f}$ registro semiótico $(m=1,2,3, \ldots)$

$\mathrm{R}^{\mathrm{m}}{ }_{\mathrm{i}}(\mathrm{A})=$ representación semiótica pésima $\left(\mathrm{i}^{\mathrm{df}}=1,2,3, \ldots\right)$ de un objeto $\mathrm{A}$ en el registro semiótico $r^{m}$

Se puede ver que si se cambia el registro semiótico cambia, necesariamente, la representación semiótica, mientras no esta dicho en viceversa; esto es, puede cambiar la representación semiótica pero manteniéndose el mismo registro semiótico.

\section{Ejemplos de representaciones semióticas de un concepto C}

\section{Concepto C}

registro semiótico $r^{1}$ : el lenguaje común representación semiótica $R^{1}{ }_{1}$ : un medio representación semiótica $\mathrm{R}_{2}^{1}$ : la mitad etc.

registro semiótico $\mathrm{r}^{2}$ : el lenguaje aritmético

representación semiótica $R_{1}^{2}: 1 / 2$ (escritura fraccionaria)

representación semiótica $R_{2}^{2}: 0.5$ (escritura decimal)

representación semiótica $\mathrm{R}_{3}^{2}: 5.10^{-1}$ (escritura exponencial) etc.

registro semiótico $\mathrm{r}^{3}$ : el lenguaje algebraico

representación semiótica $\mathrm{R}_{1}^{3}:\left\{\mathrm{xeQ}^{+} / 2 \mathrm{x}-1=0\right\}$ (escritura en la teoría de conjuntos)

representación semiótica $R_{2}^{3}: y=f(x): x \circledast x / 2$ (escritura en la teoría de funciones) etc.

registro semiótico ${ }^{4}$ : el lenguaje figural representación semiótica $\mathrm{R}^{4}{ }_{1}$ :etc.

registro semiótico $r^{5}$ : esquemas representación semiótica $R_{1}^{5}$ : 
representación semiótica $\mathrm{R}_{2}^{5}$ : representación semiótica $R_{3}^{5}$ :

etc.

etc.

Otros ejemplos pueden ser tomados de la teoría de conjuntos elemental o "ingenua", en la cual el mismo conjunto puede ser representado en varios registros semióticos y, al interno de cada uno de estos, usando varias representaciones semióticas.

\section{La paradoja de Duval y las causas de la falta de devolución}

¿En esta paradoja, así bien evidenciada por Raymond Duval, se puede esconder una potencial causa de la falta de devolución?

Según el profesor, según la noosfera y según el mismo estudiante, él (estudiante) está entrando en contacto con el "objeto" matemático pero, de hecho, y ninguno parece darse cuenta, el estudiante esta entrando en contacto solo con una particular representación semiótica de aquel "objeto". El estudiante no tiene, no puede tener, acceso directo al "objeto" y el profesor y la noosfera confunden las dos cosas; el estudiante está como bloqueado, como inhibido: no puede hacer nada mas que confundir el "objeto" con de "su representación semiótica" porque no se da cuenta, no lo sabe. Y por tanto, frente a una sucesiva necesidad conceptual, que se manifiesta por ejemplo en la necesidad de modificar la representación semiótica de aquel "objeto", el estudiante no tiene medios críticos ni culturales ni cognitivos; el profesor y la noosfera no entienden el por qué y acusan al estudiante, lo hacen responsable de alguna cosa que él no entiende.

En realidad: en esta fase paradojal, ninguno entiende qué es lo que esta sucediendo en cuanto que cada uno de los actores de esta aventura tiene una percepción diversa del problema.

\section{Semiótica y noética en el aprendizaje de la matemática}

En matemática, la adquisición conceptual de un objeto pasa necesariamente a través de la adquisición de una o más representaciones semióticas (Duval, 1988a,b,c; 1989; 1993; Chevallard, 1991; Godino, Batanero; 1994).

Aún una vez más, uso un gráfico para ilustrar la cuestión, porque me parece más incisivo y eficaz:

características de

la semiótica $\left\{\begin{array}{l}\text { representación } \\ \text { tratamiento } \\ \text { conversión }\end{array}\right.$ estas son tres actividades cognitivas diversas

Objeto $\mathrm{A}$ a representar<smiles>c1ccccc1</smiles> 
elección de las características distintivas de A

$\vartheta$

C

REPRESENTACIÓN $\mathrm{R}^{\mathrm{m}}(\mathrm{A})$ en un determinado registro semiótico $\mathrm{r}^{\mathrm{m}}$

y transformación de la representación<smiles>[R10]C1([Y20])CCCC1</smiles>

nueva representación $\left(\mathrm{i}^{1} \mathrm{j}\right) \mathrm{R}^{\mathrm{m}}{ }_{\mathrm{j}}(\mathrm{A})$ en el mismo registro semiótico $r^{\mathrm{m}}$ registro

C

transformación de registro<smiles>C1CC2CCC12</smiles>

CONVERSIÓN

nueva representación $\left(h^{1} i, h^{1} j\right) R^{n}{ }_{h}(A)$ en otro registro semiótico $r^{n}\left(n^{1} m\right)$ $(\mathrm{m}, \mathrm{n}, \mathrm{i}, \mathrm{j}, \mathrm{h}=1,2,3, \ldots)$.

Si observan las flechas, estas van, en la primera parte del gráfico, de abajo hacia arriba. Este hecho tiene una razón de ser: las características distintivas fijas del objeto A dependen de la capacidad semiótica de representación en el registro elegido. Eligiendo un registro diverso se fijarían otras características de $A$. Esto depende del hecho que dos representaciones del mismo objeto, pero en registres diversos, tienen contenidos diversos.

\section{Ejemplos de tratamiento y conversión}

$$
\text { transformación de tratamiento en } \mathrm{r}^{2}
$$

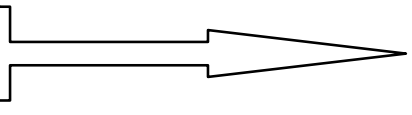

0.5

transformación de tratamiento en $r^{2}$

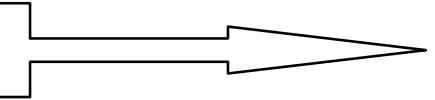

etc.

$1 / 2$
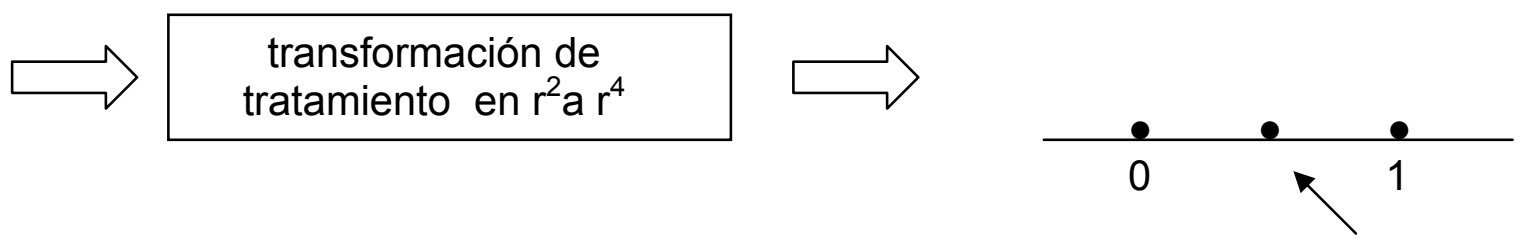

7. Características de la noética 
La adquisición conceptual de un objeto matemático se basa sobre dos de sus características "fuertes" (Duval, 1993):

- el uso de más de un registro de representación semiótica es típico del pensamiento humano

- la creación y el desarrollo de sistemas semióticos nuevos es símbolo (histórico) de progreso del conocimiento.

Estas consideraciones muestran la interdependencia estrecha entre noética y semiótica, y de como se pasa de una a la otra: no solo, por tanto, no existe noética sin semiótica, sino que la semiótica viene considerada como característica necesaria para garantizar el primer paso hacia la noética.

A este punto es necesaria una precisión sobre la teoría que de años esta desarrollando Raymond Duval.

En esta teoría él concede a la conversión un puesto central con respecto a las otras funciones, y en particular respecto a aquella del tratamiento, considerada por algunos como más decisiva desde el punte de vista del aprendizaje de la matemática.

\section{Un tentativo de "definición" de construcción}

La construcción de los conceptos matemáticos es por tanto estrechamente dependiente de la capacidad de usar más de un registro de representación semiótica de aquellos conceptos:

- de representarlos en un registro dado

- de tratar tales representaciones al interno de un mismo registro

- de convertir tales representaciones de un determinado registro en otro.

El conjunto de estos tres elementos y las consideraciones de los precedentes parágrafos ponen en evidencia la profunda relación que existe entre noética y constructivismo: ¿qué cosa quiere decir "construcción del conocimiento en matemática" si no es precisamente la unión de estas tres "acciones" sobre los conceptos, esto es la expresión misma de la capacidad de representar los conceptos (eligiendo las características consideradas distintivas), de tratar las representaciones obtenidas al interno de un registro establecido y de convertir las representaciones de un registro en otro?

Es como si estuviéramos explicando las operaciones-base que, en su conjunto, definen aquella "construcción" que, de otra forma, no sería sino un término misterioso y ambiguo, disponible a toda clase de interpretaciones, inclusive metafísicas.

\section{El fenómeno de la "escolarización" y la falta de la noética}

La renuncia de un estudiante a la devolución (obviamente inconsciente), la incapacidad del estudiante de implicarse (como resultado de éxitos negativos en los casos de tentativos efectuados), haciéndose cargo directo y personal de de la responsabilidad de la construcción del conocimiento, en el ambiente de escuela, están relacionadas con la incapacidad (en ocasiones tan solo supuesta) o de representar o de tratar o de convertir, a 
causa de una carencia didáctica específica en la base. Los profesores podrían de hecho no preocuparse de los componentes individuales de la construcción a causa de una supuesta identidad entre semiótica e noética (Duval, 1993) (identidad que es bastante difusa en el pensamiento de los profesores, especialmente entre aquellos que nunca han tenido ocasión de reflexionar sobre estas cuestiones, o que la consideran superflua; Speranza, 1997; Porlán, Rivero, Martin, 1997). Esto podría llevar a la elección de renunciar por parte del estudiante y por tanto a la escolarización de los saberes (D' Amore, 1999):

“Con el término 'escolarización de los saberes' entiendo aquí referirme a aquel acto en larga medida inconsciente, a través del cual el alumno, en un cierto punto de su vida social y escolar (pero casi siempre en el curso de la Escuela Elemental) delega a la Escuela (como institución) y al profesor de la escuela (como representante de la institución) la tarea de seleccionar para él los saberes significativos (aquellos que le son socialmente, por estatus reconocidos y legitimados de la noosfera), renunciando a hacerse cargo directo de su elección sobre la base de cualquier forma de criterio personal (gusto, interés, motivación,...). Dado que esta escolarización implica el reconocimiento del profesor como depositario de los saberes que socialmente cuentan, es también obvio que se da, mas o menos contemporáneamente, una escolarización de las relaciones interpersonales (entre estudiante y profesor y entre estudiantes y compañeros) y de las relaciones entre el estudiante y el saber: es aquello que [...] se llama "escolarización de las relaciones"'

A fin de reiterar el "juego de las ternas" (representación, tratamiento, conversión), se puede ver el resultado de la investigación descrita en D'Amore (1998). En esta investigación el mismo mensaje, concerniente a una situación conexa con un simple ejemplo de relación binaria (se daban nombres de ciudades y nombres de estados y la relación binaria era: "esta en"), venía propuesta a alumnos de varios niveles escolares en'diferentes registros semióticos y con diversas representaciones semióticas, con el objetivo de que reconocieran que se trataba, de hecho, del mismo mensaje, de la misma información.

El resultado de la investigación muestra propiamente la enorme dificultad que tienen los estudiantes

- para develar de cada representación el contenido representado

- para verificar que entre dos representaciones en un determinado registro semiótico se ha dado simplemente una transformación de representación del tipo tratamiento

- para verificar que entre dos representaciones semióticas en dos diversos registros semióticos se ha dado una transformación de tipo conversión.

Ante la falta de claves de lectura y ante la dificultad en la "lectura" de las situaciones, los estudiantes dan "sentido" al mensaje creando informaciones de varios tipos (que he llamado en algún caso "informaciones parásitas") lejanas de toda intención comunicativa del autor; y buscando agarraderos de tratamientos o conversiones en aspectos del todo marginales, como: la forma de los gráficos, el tipo de figuras casualmente presentes etc., que para el adulto son insignificantes.

\section{La falta de la devolución, la cesación de la implicación}


En caso de fracaso en el manejo de esta enorme masa de representaciones y transformaciones, es demasiado banal y simple limitarse a constatarlo, como en ocasiones parece hacer el profesor desilusionado ante la falta de aprendizaje de sus alumnos. ¿Dónde radica el motivo de tales fracasos? Ya este aspecto es mucho más interesante y un análisis de los diversos fracasos podría revelar mucho.

Pero aquí me interesa la problemática de la falta de devolución, de la cesación de una implicación personal.

Tengo en mente la figura de un estudiante considerado bueno, consciente, sensible, el cual se imita, tal vez por esta misma sensibilidad no satisfecha o por incapacidad introspectiva de la cual no tiene culpa, a observar y constatar su propio fracaso en el tentativo de hacer frente a la complejidad de la llamada en causa de la terna "representación, tratamiento, conversión". El estudiante podría decidir (también si es del todo inconsciente) de... limitar los daños aceptando el formalismo, la superficie de cuanto les viene solicitado, adaptándose a escolarizar el propio saber y el propio comportamiento, aceptando, esto es, la total mediación del profesor en dirección al objeto del saber, aceptando sus elecciones y sus gustos (D'Amore, 1999). Un análisis a profundidad de las diferentes componentes, y la capacidad de puntualizar los varios aspectos en los cuales se configura la construcción del conocimiento (en nuestro caso específico, en el ejemplo dado, aquel concepto que tiene como una de sus tantas representantes $1 / 2$ ), podría ayudar al profesor a entender cual ha sido el momento exacto de la rendición, de la falta de devolución, de la cesación de la implicación personal del estudiante en tal construcción.

Existe una gran diferencia entre la institucionalización del conocimiento por parte del profesor como representante de la institución que ha decidido cuales son los saberes que cuentan; y la escolarización, la aceptación sumisa a las elecciones del profesor.

En el primer caso el profesor tiene la tarea de ser mediador entre alumno y saber y hace del primero un ser activo: consagra las elecciones y los "descubrimientos" del alumno reconociéndole a estos un estatus institucional expendible y un permiso oficial de uso; el fundamento de todo esto está en el hecho que ha sido el alumno a construirlo.

En el segundo caso el profesor tiene la tarea de ser un mediador totalizante y hace del alumno un sujeto pasivo: les pide confianza ciega en él, les pide confianza ciega en la institución. A cambio le promete que alcanzará en su capacidad y competencia futura; pero no esta dicho sea así: la capacitad podría ser no alcanzada o podría no ser nunca expendible. El alumno cesa de construir, esto es, cesa de aprender.

Yo creo que el estudio preciso de la terna (representación, tratamiento, conversión) puede aplicarse al análisis de las situaciones de renuncia a la implicación personal, para develar el motivo que desencadenó esta renuncia, el motivo de la escolarización.

\section{BIBLIOGRAFÍA}

Chevallard Y. (1991). Dimension instrumentale, dimension sémiotique de l'acitivité mathématique. Séminaire de Didactique des Mathématiques et de l'Informatique de Grenoble. LSD2, IMAG, Université J. Fourier, Grenoble. 
D’Amore B. (1998). Oggetti relazionali e diversi registri rappresentativi: difficoltá cognitive ed ostacoli. L'educazione matemática, 1, 7-28 [texto bilingüe, italiano e inglés]. En idioma español: Uno, 15, 1998, 63-76.

D’Amore B. (1999). Scolarizzazione del sapere e delle relazioni: effetti sull' apprendimento della matemática. L'insegnamento della Matemática e delle scienze integrate. 22A, 3, 247-276. Un amplio resumen de este artículo en español se encuentra en: Resúmenes de la décimo tercera Reunión de Matemática Educativa, Santo Domingo, 1999, pág. 27.

Duval R. (1988a). Ecarts sémantiques et cohérence mathématique. Annales de Didactique et de Sciences cognitives. 1, 7-25.

Duval R. (1988b). Approche cognitive des problèmes de géométrie en termes de congruence. Annales de Didactique et de Sciences cognitives. 1, 57-74.

Duval R. (1988c). Graphiques et équations. Annales de Didactique et de Sciences cognitives. 1, 235-253.

Duval R. (1993). Registres de représentations sémiotique et fonctionnement cognitif de la pensée. Annales de Didactique et de Sciences Cognitives, ULP, IREM Strasbourg. 5, 37-65.

Duval R. (1998). Signe et object (1). Trois grandes étapes dans la problématique des rapports entre répresentation et objet. Annales de Didactique et de Sciences Cognitives. 6, 139-163.

Godino J.D., Batanero C. (1994). Significado institucional y personal de los objetos matemáticos. Recherches en Didactique des Mathématiques, 3, 325-355.

Porlán R., Rivero A., Martin A. (1997). Conocimiento profesional y epistemológico de los profesores. Enseñanza de las Ciencias, 15-2, 155-171.

Speranza F. (1997). Scritti di Epistemologia della Matematica. Bologna, Pitagora. 